\title{
Can Sedentary and Stationary Time be Accurately Assessed With Wrist- and Hip-Worn Accelerometers?
}

\author{
Patty Freedson \\ University of Massachusetts, Amherst
}

In the past, a self-report measure of sitting time, TV time, and/or computer time was the method typically used to assess sedentary time. More recently, wearable accelerometers have become the method of choice to improve measurement accuracy and precision. Numerous prediction methods are available to translate device output into estimates of sedentary time. In this issue of the Journal for the Measurement of Physical Behaviour (JMPB), we highlight the article by Florez-Pregonero and colleagues entitled, "The Accuracy of the Placement of Wearable Monitors to Classify Sedentary and Stationary Time Under Free-living Conditions." This study was conducted by Dr. Florez-Pregonero when he was a doctoral student at Arizona State University under the mentorship of Dr. Barbara Ainsworth. The findings of this study provide evidence of limitations in the accuracy of sedentary and stationary time algorithms from accelerometers worn on the hip and wrist. The authors also show that a device algorithm detecting posture position is important to differentiate sedentary and stationary behaviors.

Dr. Florez-Pregonero agreed to be interviewed about this article and his responses to several questions appear below. A commentary reacting to the study design and findings is then provided by Dr. Alex Rowlands (alex.rowlands@leicester.ac.uk), an Associate Editor for JMPB and a Senior Research Fellow at the University of Leicester in England.

\section{Interview With Alberto Florez-Pregonero, PhD, Associate Professor, Pontificia University}

When developing or analyzing wearable device output to estimate sedentary time and stationary time, it is critical to use methods that are robust. Elaborate on your rationale for selecting direct observation as the criterion for evaluating device performance?

Direct observation allows the identification of several sedentary behavior characteristics. For example, direct observation allows one to identify not only the time a given behavior occurs (such as stationary or sedentary time), but it identifies contextual information such as when, where, and with whom the behaviors under observation occur.

What were the challenges you experienced with data collection and analysis?

Because of the large amount of information collected, direct observation is labor intensive. We collected free-living data, which

Freedson (psf@kin.umass.edu) is Editor-in-Chief of the Journal for the Measurement of Physical Behaviour, and Professor Emerita, Dept. of Kinesiology, University of Massachusetts, Amherst. brought to us some extra logistical challenges. Beside the normal aspects of an observation session (i.e., initializing monitors, downloading) we needed to meet the participants in their usual environment, which added additional logistical complications for the researchers and the participants. We were required to minimize our interactions with participants and merge ourselves into their normal life without disruption. Also, we found ourselves following our participants in different types of situations that were challenging (e.g., hiking, biking, etc.).

Do you think that our current device algorithms for estimating sedentary time and stationary time are adequate for large scale surveillance and intervention studies?

Current device algorithms provide important information that are useful for tracking population-level activity levels and the efficacy of interventions. However, there are still a number of questions about how sedentary behavior should be defined and this is likely to evolve over time. Measurement scientists are then challenged to develop new algorithms and approaches to address these changing definitions. We suspect this is going to be a continuous challenge and surveillance measures should consider adopting these new approaches while retaining older approaches for long-term monitoring. Intervention studies should focus on measuring the behavior that is being targeted (e.g., stationary time vs. seated posture) as precisely as possible.

What are the next steps to advance the assessment of sedentary time and stationary time using wearable devices?

Activity identification using machine-learning approaches is an important focus of many labs around the world. However, as sedentary and related behaviors have important context-specific forms and purposes, understanding how context may be inferred or combined with activity information in a more meaningful way is an important next step to enhance our assessment of sedentary time.

\section{Commentary by Alex Rowlands, PhD, Senior Research Fellow, Diabetes Research Centre; NIHR Leicester Biomedical Research Centre, University of Leicester}

Accelerometers are widely used to assess physical behaviors in large-scale surveys including UK Biobank and the US National Health and Nutrition Examination Survey (NHANES). Given that sedentary behavior is an established risk factor for chronic disease and mortality (Biswas et al., 2015), it is important to be able to establish whether we can quantify sedentary time from these accelerometer data. This article by Dr. Florez-Pregonero and colleagues 
highlighted that cut-points methods that are widely used have limited accuracy in assessing sedentary time in free-living settings.

Sedentary time is defined as time spent in behaviors with a low waking energy expenditure ( $>1.5$ METs) while in a lying, reclining, or seated posture (Tremblay et al., 2017). As the authors of this article point out, despite cut-points reflecting movement and not posture, they are frequently used to estimate sedentary time (e.g. Matthews et al., 2008). This is most likely due to their ease of implementation compared to more sophisticated approaches that try to distinguish between postures (e.g. Kerr et al., 2018; Rowlands et al., 2014).

In contrast, stationary time refers to time spent in waking behaviors that are non-ambulatory, with no caveats for posture or energy expenditure (Tremblay et al., 2017). When simply looking at magnitudes of acceleration (i.e., movement), as cut-points do, outcomes are likely approximating stationary time rather than sedentary time. For example, in a laboratory-based protocol, Bakrania et al. (2016) showed that hip- and wrist-worn raw acceleration cut-points showed excellent ability for separating stationary behaviors from light-intensity activities, but relatively poor ability to separate sedentary behaviors from light-intensity activities.

In their study, Florez-Pregonero and colleagues set out to determine whether this was the case in free-living data for two wear-sites (hip and wrist) and two widely deployed monitors (ActiGraph and GENEActiv) used in accelerometer studies. A major strength of the study is the free-living protocol with direct observation as the criterion. Evaluating the accuracy of methods under the conditions they will be deployed in gives insight into what the 'real world' accuracy will most likely be.

The limited accuracy reported for the cut-point approaches (for all monitors and wear-sites) to assess both sedentary time and stationary time is a cautionary note to researchers applying cutpoints. Not surprisingly, given the inability of cut-point-based analyses to account for posture, sedentary time assessed by hipworn accelerometers tended to be over-estimated. The converse was true for wrist-worn accelerometers, most notably by the ActiGraph. The ActiGraph cut-points deployed for the wrist were developed for hip-worn monitors, so this is a clear illustration of the importance of using cut-points at the wear-site for which they were developed.

When the authors developed wear-site specific cut-points, sedentary time was generally over-estimated and stationary time under-estimated, with overall lower percent error for stationary time. However, kappa values, sensitivity, and specificity indicated agreement was still relatively poor. Given cut-points are applied to the magnitude of accelerations recorded, it is possible that cut-points can be developed that will improve the performance of classifying stationary time, but perhaps less likely that this will extend to sedentary time.

More sophisticated approaches that use machine learning methods to classify sitting time (e.g. Kerr et al., 2018), or consider arm elevation from wrist-worn accelerometer data, show higher agreement, relative to the activPAL, for measures of sedentary time (Rowlands et al., 2014). The challenge is to make these approaches as accessible to researchers as cut-point approaches, thus facilitating more accurate classification of time spent in sedentary behaviors in large-scale surveys.

The authors have generated a valuable dataset that is comprehensively labeled. It would be possible to use this dataset to assess the accuracy of other cut-points aiming to classify stationary time (e.g. Bakrania et al., 2016), and to evaluate machine learning approaches that aim to classify sedentary time. Given the detailed nature of the observation coding employed by the authors, it would also be possible to evaluate the accuracy of machine learning approaches aiming to classify type of activities. Moving forward, the generalizability of results could be increased by assessing accuracy of analytical approaches to accelerometer data across multiple study sites that have labeled free-living data (and/or concurrent activPAL data for sedentary time and stationary time). As outlined by John (2018), this could be made possible through access to raw data via data-sharing platforms, a practice that is common in engineering and computer science.

But, as the authors point out, the cut-point approach is the one that is most likely to be widely implemented at present. Given this, and the findings reported by the authors, it is perhaps pertinent to ensure that when using cut-point approaches to analyze accelerometer data: a) the most accurate wear-site specific cut-point is used; and b) the term 'sedentary time' is not used and, instead, the term 'stationary time' is used.

\section{References}

Bakrania, K., Yates, T., Rowlands, A.V., Sanders, J., Esliger, D.W., Bunnewell, S., . . . Edwardson, C. (2016). Developing and validating intensity-based thresholds on raw accelerometer data for discriminating between sedentary behaviours and light-intensity physical activities: a MAD approach. PLoS One, 11(10): e0164045. PubMed ID: 27706241 doi:10.1371/journal.pone.0164045

Biswas, A., Oh, P.I., Faulkner, G.E., Bajaj, R.R., Silver, M.A., Mitchell, M.S., \& Alter, D.A. (2015). Sedentary time and its association with risk for disease incidence, mortality, and hospitalization in adults: a systematic review and meta-analysis. Annals of Internal Medicine, 162(2), 123-132. PubMed ID: 25599350 doi:10.7326/M14-1651

John, D. (2018). Commentary: Validation of a popular consumer activity tracker. Journal for the Measurement of Physical Behaviour, 1, 3.

Kerr, J., Carlson, J., Godbole, S, Cadmus-Bertram, L, Bellettiere, J, \& Hartman, S. (2018). Improving hip-worn accelerometer estimates of sitting using machine learning methods. Medicine \& Science in Sports \& Exercise, 50, 1518-1524. PubMed ID: 29443824 doi:10.1249/ MSS.0000000000001578

Matthews, C., Chen, K.Y., Freedson, P.S., Buchowski, M.S., Beech, B.M., Pate, R.R., \& Troiano, R.P. (2008). Amount of time spent in sedentary behaviors in the United States, 2003-2004. American Journal of Epidemiology, 167(7), 875-881. PubMed ID: 18303006 doi:10.1093/ aje/kwm390

Rowlands, A.V., Olds, T.S., Hillsdon, M., Pulsford, R., Hurst, T.L., Eston, R.G., . . . Langford, J. (2014). Assessing sedentary behaviour with the GENEActiv: Introducing the Sedentary Sphere. Medicine \& Science in Sports \& Exercise, 46, 1235-1247. PubMed ID: 24263980 doi:10.1249/MSS.0000000000000224

Tremblay, M., Aubert, S., Barnes, J.D., Saunders, J., Carson, V., Latimer-Cheung, A.E., . . . Chinapaw, M.J.M. (2017). Sedentary Behavior Research Network (SBRN) - Terminology Consensus Project process and outcome. International Journal of Behavioral Nutrition and Physical Activity, 17(75), 1-17. doi:10.1186/s12966017-0525-8 